\title{
Thermographic modeling of pollution of reservoirs with solutions $\mathrm{NaCl}$
}

\author{
Konstantin Bozhko ${ }^{1}$, Volodymyr Maslov $^{2}$, Volodymyr Porev ${ }^{1}$, Volodymyr Timofeev ${ }^{1}$, Evgen Venger ${ }^{2}$, \\ Vadim Dunaevskiy ${ }^{2}$, Vitaliy Kotovskiy ${ }^{1}$, Svetlana Nazarchuk ${ }^{1}$, Mykhailo Lysenko ${ }^{1}$ \\ ${ }^{1}$ National Technical University of Ukraine, "Kyiv Polytechnic Institute", Kyiv, Ukraine \\ ${ }^{2} \mathrm{~V}$. Lashkaryov Institute of Semiconductor Physics NAS of Ukraine, Kyiv, Ukraine
}

Email address:

maslov@isp.kiev.ua (V. Maslov)

\section{To cite this article:}

Konstantin Bozhko, Volodymyr Maslov, Volodymyr Porev, Volodymyr Timofeev, Evgen Venger, Vadim Dunaevskiy, Vitaliy Kotovskiy, Svetlana Nazarchuk, Mykhailo Lysenko. Thermographic Modeling of Pollution of Reservoirs with Solutions NaCl. American Journal of Environmental Protection. Vol. 3, No. 5, 2014, pp. 263-266. doi: 10.11648/j.ajep.20140305.19

\begin{abstract}
A thermographic method for studying the dynamics of the process of pollution of the reservoirs with solutions containing salt has been approved. The method enables control over the stratification of polluted water with a contaminative solution by its thermal field, regardless of the transparency of the components of pollution. Authors have offered the analytical solution for the model of running of the spot of a pollutive solution over the bottom of the tank, which can greatly simplify calculations. The result of research is confirmation of the hypothesis of the predominant influence of the mechanism of a gravitational transfer when the reservoirs are being contaminated with salt solutions. Pollution is localized in the bottom area of a pond. The water intake for the needs of cities and towns should be performed only at surface layers of the reservoirs because many soluble contaminants concentrate in bottom layers.
\end{abstract}

Keywords: Thermographic Method, Pollution, Water

\section{Introduction}

During the study of the process of water pollution by the discharge of industrial and municipal wastes its dynamics and particularly the stratification of polluted water and its motion raise an important question because the "health" of a reservoir depends on the activity of dynamic processes. Numerical methods of stratified fluid dynamics are the object of many investigations, particularly [1-5].

Contaminant components often have a higher temperature relative to the temperature of the reservoir. In this case, it is necessary to deal with the issue of a heat transfer. Numerical methods for solving problems of a heat transfer in the liquid have been examined in various works, for example, in [6].

Thermography, as a method for the remote measurement of a thermal field, enables the physical modeling of the process of pollution and the stratification of contaminated water in particular. Heavy pollutive components have greater density than water, therefore they settle to the bottom in the accumulation zone of the reservoir. If a pond is streamless and the discharge has a higher temperature than water in the pond, the following processes of the simultaneous transfer of the discharge along three mechanisms occur:

- gravitational mass transfer (settling to the bottom);

- diffusion through the interface between the discharge and water of the reservoir;

- conductive heat transfer through the interface (no convection).

The gravitational transfer is the main factor in the proposed model. The general theory of the flow of a drop of liquid under the action of a gravitational force has been examined in [7]. Unlike an analytical solution [7] to the problem of running of a spherical drop of liquid over a solid surface, we take account of temperature dependence of the parameters of liquid. At the same time, we disregard the diffuse process because its speed is inferior to the speed of a gravitational transfer and the rate of a heat transfer.

The purpose of modeling is to find an analytical solution to the problem of a mass transfer, when polluting the streamless reservoir, in consideration of the temperature of the contaminants. Experimental confirmation of the model should be thermograms of the stratified liquid in which a heated saline solution serves as contaminants. The solution has been discharged into the aquarium, as the model environment. 


\section{Experiment Results}

We have done research on the process of distribution of the $20 \%$ saline solution $\mathrm{NaCl}$ heated to a temperature of $60^{\circ} \mathrm{C}$ when contaminating the closed reservoir (aquarium with fresh water) with it.

The raised temperature of the saline solution made it possible to study the distribution of the solution in the aquarium, using thermography. The selection of the above salt can be explained by the fact that over the winter it is the main component, which is often used by public utilities for melting ice and snow in Ukrainian cities, towns and villages. Ultimately, this salt mixture or similar salts fall into a natural reservoir.

In the course of our experiments, the temperature of water in the tank was $20^{\circ} \mathrm{C}$. The solution was injected at the front (control) side of the aquarium. Since the solution is transparent, it can be identified in water by the thermal field only. The form taken by a saline solution in the tank was controlled by the thermal field on the side of the aquarium. In this case, the front side of the aquarium is the plane $\mathrm{XOZ}$, which halves space with the area of contamination (Figure 1). If we remove the front side (push it to a viewer), we get a problem for the real reservoir without sides. Thus, the thermal field in half space bounded by the plane $\mathrm{XOZ}$ is the solution to the problem of temperature distribution (i.e. the distribution of contaminants) for the unlimited reservoir when adding its mirror image to half space, which is relative to this plane.

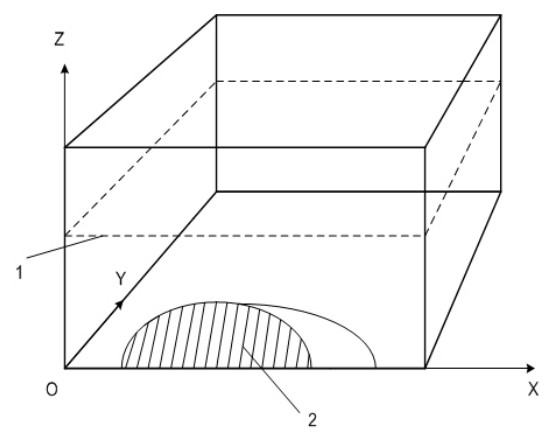

Figure 1. Schematic representation of the aquarium with water and the location of a contaminated substance at the bottom of it (1 - water level, 2 cross section of the area of contamination in the plane XOZ).

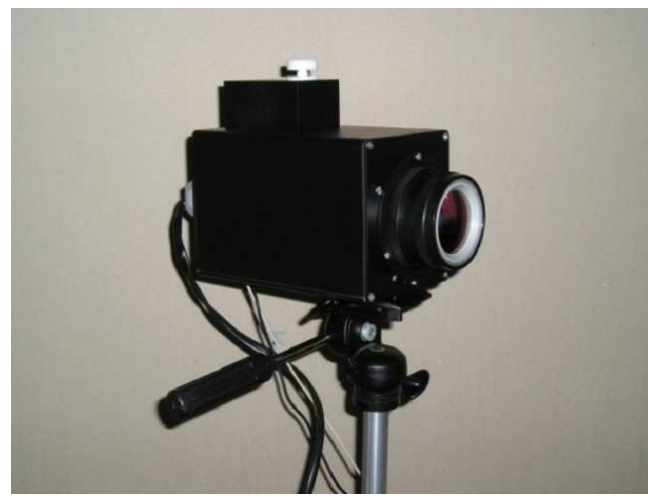

Figure 2. Thermograph

The use of glass, which is transparent in the infrared range, for the front side of the aquarium is a prerequisite for the experiment. As a rule, this is quartz glass.

Infrared thermography (see Fig. 2) is based on a photodetector camera and a personal computer, and it has the following characteristics:

- matrix of the camera has a working area of $256(\mathrm{H}) \times 290$ (V) pixels;

- the range of spectral sensitivity of the camera is within 2 to 5.3 microns;

- temperature sensitivity is $0.07^{\circ} \mathrm{C}$;

- the matrix is cooled by liquid nitrogen $(0.8 \mathrm{~L}$. for 6 hours of uninterrupted operation).

During the experiment we have obtained thermograms of the tank in relation to the time of observation; specifically, at the start of running of the saline solution over the bottom of the tank (Fig. 3) and at intervals of 600 (Fig.4), 1,200 (Fig. 5) and 1,800 seconds (Fig. 6) after the solution started running over the tank's bottom.

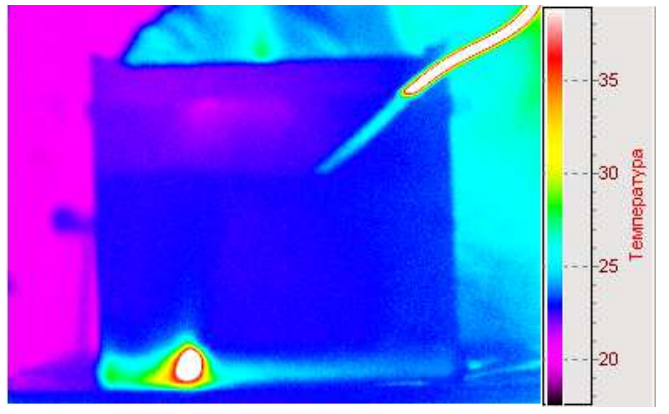

Figure 3. Thermogram of the aquarium with water at the start of running of the saline solution over the bottom; the solution's temperature at the center of pollution $t=50.07^{\circ} \mathrm{C}$

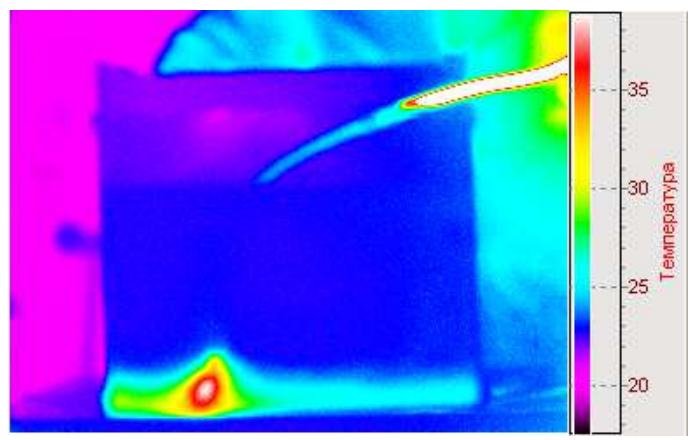

Figure 4. Aquarium's thermogram made in $600 \mathrm{~s}$. after the solution started running; $t=40.62^{\circ} \mathrm{C}$

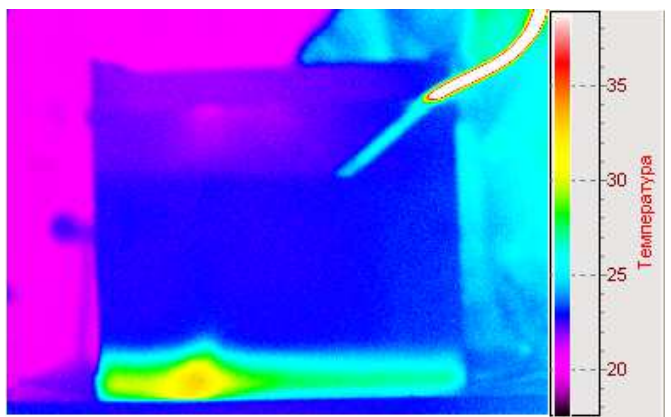

Figure 5. Aquarium's thermogram made in 1,200 s. after the solution started running; $t=36.28^{\circ} \mathrm{C}$ 


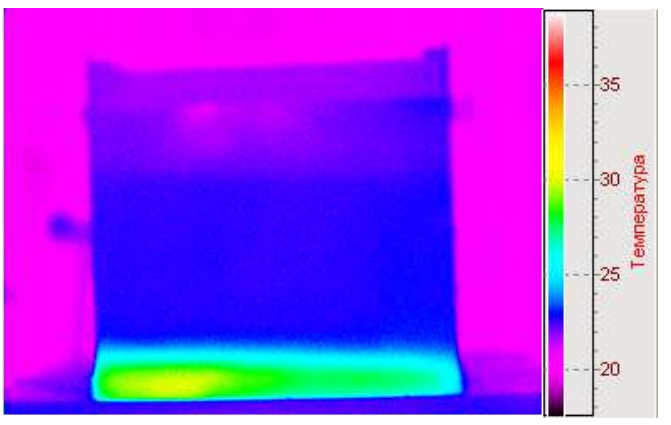

Figure 6. Aquarium's thermogram made in 1,800 s. after the solution started running; $t=33.38^{\circ} \mathrm{C}$.

When injecting the saline solution, it takes a spherical shape (see Fig. 3). This can be explained by the action of surface tension forces. Initially, the large drop is not deformed by gravity, as it falls freely. The resultant of the forces of gravity and Archimedes is low because of a slight difference in density of water and the solution, therefore a heavier solution is slowly sinking. The deformation of a spherical drop begins only when it reaches the bottom of the tank (see Fig. 4).

These thermograms show that at first the speed of running of the salt solution (the speed of deformation of a sphere) over the bottom of the aquarium is lower than at final phases of the process (Fig.5 and Fig.6). This can be explained by thermal expansion of the solution whereby its density is lower at the start of running and, consequently, the resultant of the forces of gravity and Archimedes, which acts on the spherical drop, is lower as well. When cooling the solution, its density increases and the spreading process accelerates. The effect of solution's viscosity-temperature relationship on this process is very little and we can disregard it. It is worth adding that the process of a heat transfer depends on the temperature gradient and the area of an interface between water and the solution. The area of the interface increases when the solution is spreading, which is the growth factor of a heat transfer. However, the temperature gradient decreases that slows down the process. The thermal field of the solution and adjacent layer of water (see Fig. 5 and Fig. 6) is forming under the influence of these antipodal factors.

\section{The Mathematical Model of the Process of Running of the Saline Solution $\mathrm{NaCl}$ in the Water Environment (over the Bottom of the Tank)}

When a spot of the saline solution is running over the bottom of the tank (Figure 7), its radius varies with time according to the law of the $1 / 4$ degree [7].

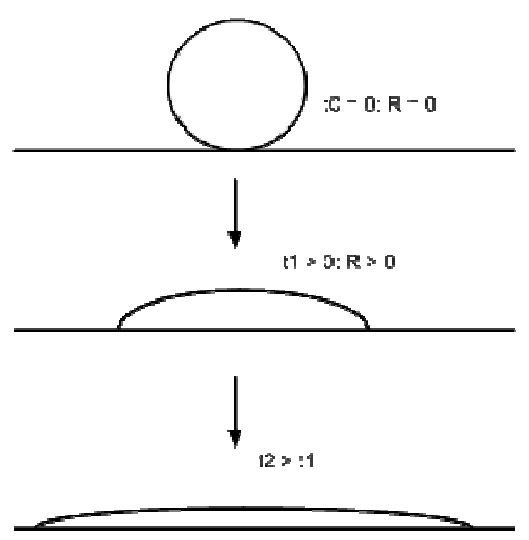

Figure 7. Change in the radius $R$ of the spot of the solution at the bottom of the aquarium in the course of time $t$

Unlike the previous theory of the flow of a drop [7], it is necessary to consider a force pushing the solution out of water (Archimedes' force). To do so, instead of the mass of a drop in a basic equation (1) we insert a difference in the mass of a saline solution and the water, which has the same volume as the solution has. Given the above, dependence of the radius $\mathrm{R}$ of a spot on the time $t$ is:

$$
\mathrm{R}=(6 \Delta \mathrm{m} \Delta \sigma / \pi \chi \eta \rho) 1 / 4 \mathrm{t}^{1 / 4}
$$

where $\Delta \mathrm{m}$ is a difference in the mass of the saline solution and water of the same volume, $\Delta \sigma$ is the difference of surface stress at the water - saline solution interface; $\eta$ is viscosity of the saline solution; $\rho$ is density of the saline solution; $\chi$ is a correction factor.

This dependence is well matched with the partial solution of the Navier-Stokes equation for running of a drop of liquid over a surface [8].

The saline solution whose volume totals $\mathrm{V}=50 \mathrm{ml}$ has been injected into the aquarium. The mass of water of the same volume is $50 \mathrm{~g}$ and density of the $20 \%$ solution $\mathrm{NaCl}$ is $\rho=$ $1,148 \mathrm{~kg} / \mathrm{m}^{3}$ [9]. The mass of the solution is $\mathrm{mp}=\rho \mathrm{v}$ or $\mathrm{mp}=$ $0.0574 \mathrm{~kg}$. A difference in the mass of the solution and water, disregarding thermal expansion is $\Delta \mathrm{m} 0=0.0574-0.05=$ $0.0074 \mathrm{~kg}$. The coefficient of volumetric thermal expansion of water has the average value $\alpha=4.5 \cdot 10^{-4} \mathrm{~K}^{-1}[5]$ that gives the coefficient of a reduction in mass $\mathrm{k}_{\mathrm{m}}=1+\alpha \dot{\alpha}$ or $\mathrm{k}_{\mathrm{m}}=1+4.5$ - $10^{-4} \mathrm{~K}^{-1} 30 \mathrm{~K}=1.0135$ for the temperature difference $\Delta \mathrm{T}=30$ $\mathrm{K}$. Then the difference in mass is $\Delta \mathrm{m}=\Delta \mathrm{m} 0 / \mathrm{k}_{\mathrm{m}}$ or $\Delta \mathrm{m}=$ $0.0074 / 1.0135=0.0073 \mathrm{~kg}$. To calculate the difference of drops when they are spreading, we take reference values of the coefficient of surface stress [10]: the coefficient of surface stress for clear water at a temperature of $20^{\circ} \mathrm{C}$ is equal to $0.073 \mathrm{~N} / \mathrm{m}$ [9], the coefficient of surface stress for a $20 \%$ saline solution is $0.075 \mathrm{~N} / \mathrm{m}$ for a temperature of $50^{\circ} \mathrm{C}$, a difference in the coefficients of surface stress is $\Delta \sigma=0.0075$ $0.0073==0.002 \mathrm{~N} / \mathrm{m}$. The viscosity of a $20 \%$ saline solution is $1.5 \mathrm{MPa} \bullet \mathrm{s}$ [10]. The correction factor is $\chi=10$ [7].

In accordance with calculations done by the formula (1) a graph showing dependence of the radius of pollution on the time has been plotted (see Fig. 8). 


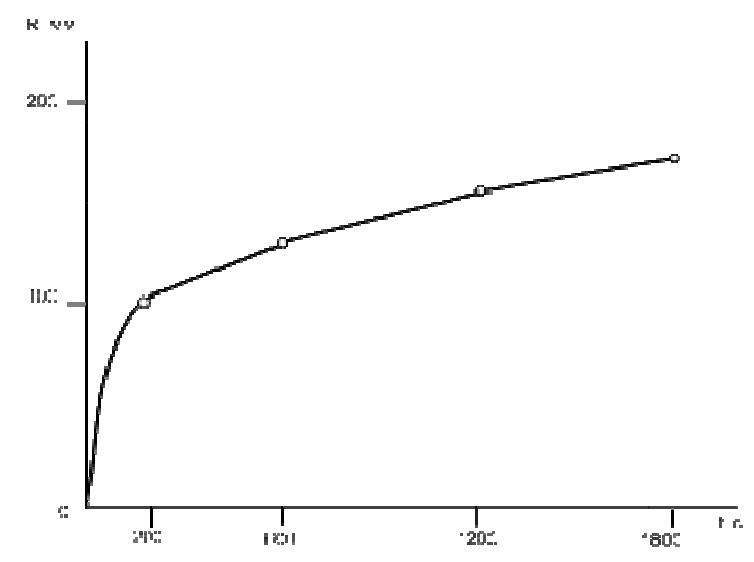

Figure 8. Dependence of the calculated radius $R$ of a spot on the time $t$

A time lag of a change in the radius of pollution shown on the graph (Figure 8) behind thermograms (Figures 4 - 6) can be explained by the lower rate of a heat transfer, compared with the speed of a gravitational transfer (running of the drop). Thermograms give us information on the distribution of the thermal field whose change is different from the process of a mass transfer.

\section{Discussion}

The results of research allow us to point out that the proposed method for visualizing the spread of transparent solutions of inorganic salts (contaminants) in water supposes preheating of these pollutive solutions prior to their discharge in water. Moreover, preheating is supposed to conduce to the convection and mixing of an injected contaminative solution. However, it is experimentally established that even the contaminative solution, which has been heated to a higher temperature, gravitates to the bottom, as it has a greater density, and covers gradually a part of the bottom of a reservoir. The obtained results are original and their topicality can be explained by the fact that over the winter water-soluble salts are used by public utilities for removing a glaze of ice from sidewalks and roads. When snow melts, in time the contaminants will fall into reservoirs. And our research has shown that the contaminants settle to the bottom and block the microorganisms, which are supposed to purify reservoirs.

Thus this research suggests that instead of water-soluble reagents it is necessary to use the fine gravel, which could be collected in spring for reuse in the next wintertime.

\section{Conclusions}

1. A thermographic method for studying the dynamics of the process of pollution of the reservoirs with solutions containing salt has been approved. The method enables control over the stratification of polluted water with a contaminative solution by its thermal field, regardless of the transparency of the components of pollution.

2. We have offered the analytical solution for the model of running of the spot of a pollutive solution over the bottom of the tank, which can greatly simplify calculations.

3. The result of research is confirmation of the hypothesis of the predominant influence of the mechanism of a gravitational transfer when the reservoirs are being contaminated with salt solutions. Pollution is localized in the bottom area of a pond. The water intake for the needs of cities and towns should be performed only at surface layers of the reservoirs because many soluble contaminants concentrate in bottom layers.

\section{References}

[1] S.S. Kuzikov "The methods of numerical calculation of problems of stratified fluid flow," News of the Altai State University. Mathematics. No.1 (53). - Barnaul, 2007 - pp. 50 51.

[2] S. Li, Y. Zhuang, L. Zhang, Y. Du, and H. Liu. "Worldwide performance and trends in nonpoint source pollution modeling research from 1994 to 2013: A review based on bibliometrics," Journal of Soil and Water Conservation, 2014, 69(4), pp. 121A-126A.

[3] M.B. McGechan, D.R. Lewis, A.J.A. Vinten "A river water pollution model for assessment of best management practices for livestock farming" Biosystems Engineering -Volume 99, Issue 2, February 2008, pp. 292-303.

[4] R. D. Morgenstern "Economic Analyses at EPA: Assessing Regulatory Impact” Routledge, 2014, pp. 496.

[5] Jia-Qian Jiang, Barry Lloyd "Progress in the development and use of ferrate (VI) salt as an oxidant and coagulant for water and wastewater treatment" Water Research, V. 36, Is. 6, 2002, pp. 1397-1408.

[6] S. Patankar "Numerical methods for solving problems of heat transfer and fluid dynamics."M. Energoatomizdat, 1984 - p. 124.

[7] Y. Goryunov Physico-chemical regularities of the distribution of liquid metal on a solid metal surface // Successes of Chemistry, Volume XXXIII, Issue 9 - 1963 - pp. 1062 - 1083.

[8] M.A. Ponomareva, G.R. Schrager, V.A. Yakutenok Using the Dupre-Young equation to solve the problem of spreading of liquids with limited wetting // Bulletin of the Tomsk State University. Mathematics and Mechanics. 2008. No.1 (2). pp. 90-96.

[9] A.I. Volkov, I.M. Zharskiy "Large Chemical Directory", M.: Modern School, 2005, - p. 608.

[10] A.A. Ravdel Concise Reference Book of Physic-Chemical Values. Eighth Edition, Edited by A.A. Ravdel and M.A. Ponomareva, L .: Chemistry, 1983 - p. 144. 